\title{
Operator Fractional Brownian Motion and Martingale Differences
}

\author{
Hongshuai Dai, ${ }^{1,2}$ Tien-Chung $\mathrm{Hu}^{3}{ }^{3}$ and June-Yung Lee ${ }^{3}$ \\ ${ }^{1}$ School of Statistics, Shandong University of Finance and Economics, Jinan 250014, China \\ ${ }^{2}$ College of Mathematics, Guangxi University, Nanning 530004, China \\ ${ }^{3}$ Department of Mathematics, National Tsing-Hua University, Hsinchu 30043, Taiwan
}

Correspondence should be addressed to Hongshuai Dai; daihongshuai@gmail.com

Received 31 July 2014; Accepted 4 September 2014; Published 27 October 2014

Academic Editor: Yong Ren

Copyright (C) 2014 Hongshuai Dai et al. This is an open access article distributed under the Creative Commons Attribution License, which permits unrestricted use, distribution, and reproduction in any medium, provided the original work is properly cited.

\begin{abstract}
It is well known that martingale difference sequences are very useful in applications and theory. On the other hand, the operator fractional Brownian motion as an extension of the well-known fractional Brownian motion also plays an important role in both applications and theory. In this paper, we study the relation between them. We construct an approximation sequence of operator fractional Brownian motion based on a martingale difference sequence.
\end{abstract}

\section{Introduction}

Fractional Brownian motion (FBM) is a continuous Gaussian process with stationary increments. It is one of the wellknown self-similar processes. Some studies of financial time series and telecommunication networks have shown that this kind of processes with long-range dependency memory might be a better model in some cases than the traditional standard Brownian motion. Due to its applications in the real world and its interesting theoretical properties, fractional Brownian motion has become an object of intense study. One of those studies concerns obtaining its weak limit theorems; see, for example, Enriquez [1], Nieminen [2], Sottinen [3], Li and Dai [4], and the references therein.

Based on the study of FBMs, many authors have proposed a generalization of it and have obtained many new processes. An extension of FBMs is the operator fractional Brownian motion (OFBM). OFBMs are multivariate analogues of onedimensional FBMs. They arise in the context of multivariate time series and long range dependence (see, e.g., Chung [5], Davidson and de Jong [6], Dolado and Marmol [7], Robinson [8], and Marinucci and Robinson [9]). Another context is that of queuing systems, where reflected OFBMs model the size of multiple queues in particular classes of queuing models. They are also studied in problems related to, for example, large deviations (see Delgado [10] and Konstantopoulos and Lin [11]). Similar to those for FBMs, weak limit theorems for OFBMs have been studied recently. Some new results on approximations of OFBMs have been obtained. See Dai $[12,13]$ and the references therein.

It is well known that a martingale difference sequence is extremely useful because it imposes much milder restrictions on the memory of the sequence than under independence, yet most limit theorems that hold for an independent sequence will also hold for a martingale difference sequence. In recent years, some researchers have used this type of sequences to construct approximation sequences of some known processes. For example, Nieminen [2] studied the limit theorems for FBMs based on martingale difference sequences. This is a natural motivation for this paper. The direct motivation is the recent works by Dai $[12,13]$, in which, based on a sequence of IID random variables, the author presented some weak limit theorems for some special kinds of OFBMs.

In this short paper, we establish a weak limit theorem for a special case of OFBMs, which comes from Maejima and Mason [14], however, based on martingale difference sequences. The rest of this paper is organized as follows. In Section 2, we recall OFBMs and martingale-difference sequences and present the main result of this paper. Section 3 is devoted to prove the main result of this paper. 


\section{Operator Fractional Brownian Motion and Martingale Differences}

In this section, we first introduce a special type of OFBMs. Let $\operatorname{End}\left(\mathbb{R}^{d}\right)$ be the set of linear operators on $\mathbb{R}^{d}$ (endomorphisms) and let Aut $\left(\mathbb{R}^{d}\right)$ be the set of invertible linear operators (automorphisms) in $\operatorname{End}\left(\mathbb{R}^{d}\right)$. For convenience, we do not distinguish an operator $D \in \operatorname{End}\left(\mathbb{R}^{d}\right)$ from its associated matrix relative to the standard basis of $\mathbb{R}^{d}$. As usual, for $c>0$,

$$
c^{D}=\exp ((\log c) D)=\sum_{k=0}^{\infty} \frac{1}{k !}(\log c)^{k} D^{k} .
$$

Throughout this paper, we use $\|x\|$ to denote the usual Euclidean norm of $x \in \mathbb{R}^{d}$. Without confusion, for $A \in$ $\operatorname{End}\left(\mathbb{R}^{d}\right)$, we also let $\|A\|=\max _{\|x\|=1}\|A x\|$ denote the operator norm of $A$. It is easy to see that, for $A, B \in \operatorname{End}\left(\mathbb{R}^{d}\right)$,

$$
\|A B\| \leq\|A\| \cdot\|B\|
$$

and, for every $A=\left(A_{i j}\right)_{d \times d} \in \operatorname{End}\left(\mathbb{R}^{d}\right)$,

$$
\max _{1 \leq i, j \leq d}\left|A_{i j}\right| \leq\|A\| \leq d^{3 / 2} \max _{1 \leq i, j \leq d}\left|A_{i j}\right| \text {. }
$$

Let $\sigma(A)$ be the collection of all eigenvalues of $A$. We let

$$
\begin{aligned}
& \lambda_{A}=\min \{\operatorname{Re} \lambda: \lambda \in \sigma(A)\}, \\
& \Lambda_{A}=\max \{\operatorname{Re} \lambda: \lambda \in \sigma(A)\} .
\end{aligned}
$$

Let $x^{\prime}$ denote the transpose of a vector $x \in \mathbb{R}^{d}$. We now extend the fractional Brownian motion of Riemann-Liouville type studied by Lévy [15, page 357] to the multivariate case.

Definition 1. Let $D$ be a linear operator on $\mathbb{R}^{d}$ with $1 / 2<\lambda_{D}$, $\Lambda_{D}<1$. For $t \in \mathbb{R}_{+}$, define

$$
X(t)=\int_{0}^{t}(t-u)^{D-I / 2} d W(u)
$$

where $W(u)=\left\{W^{1}(u), \ldots, W^{d}(u)\right\}^{\prime}$ is a standard $d$ dimensional Brownian motion. We call the process $X=$ $\{X(t)\}$ an operator fractional Brownian motion of RiemannLiouville (RL-OFBM).

As is standard for the multivariate context, we assume that RL-OFBM is proper. A random variable in $\mathbb{R}^{d}$ is proper if the support of its distribution is not contained in a proper hyperplane of $\mathbb{R}^{d}$.

Remark 2. The operator fractional Brownian motion in the current work is a special case of the operator fractional Brownian motions in the work of Maejima and Mason [14, Theorem 3.1].

Remark 3. The RL-OFBM $X$ defined by (5) is an operator selfsimilar Gaussian process.
In this short paper, we want to obtain an approximation of the RL-OFBM $X$. Inspired by Nieminen [2], we want to construct an approximation sequence of RL-OFBM $X$ by martingale differences.

Let $\left\{\xi^{(n)}=\left(\xi_{i}^{(n)}, \mathscr{F}_{i}^{n}\right)_{1 \leq i \leq n}\right\}_{n \in \mathbb{N}}$ be a sequence of square integrable martingale differences such that for every sequence $\left\{i_{n}\right\}$ with $\lim _{n \rightarrow \infty} i_{n}=\infty$, where $1 \leq i_{n} \leq n$,

$$
\begin{aligned}
& \lim _{n \rightarrow \infty} \frac{\left(\xi_{i_{n}}^{(n)}\right)^{2}}{1 / n}=1, \quad \text { a.s., } \\
& \max _{1 \leq i \leq n}\left|\xi_{i}^{(n)}\right| \leq \frac{C}{\sqrt{n}}, \quad \text { a.s., }
\end{aligned}
$$

for some $C \geq 1$. [16].

The following lemma follows from Jacod and Shiryaev

Lemma 4. Under condition (7) and the condition

$$
\sum_{i=1}^{\lfloor n t\rfloor}\left(\xi_{i}^{(n)}\right)^{2} \longrightarrow t, \quad \text { a.s. }
$$

the processes

$$
B^{n}(t)=\sum_{i=1}^{\lfloor n t\rfloor} \xi_{i}^{(n)}
$$

converge in distribution to a Brownian motion $B$, as $n \rightarrow \infty$.

Remark 5. Such a type of sequences is very useful, since it is very easy to obtain it in the real world. See, for example, Nieminen [2].

Below, we extend Lemma 4 to the $d$-dimensional case. Define

$$
\eta_{i}^{(n)}=\left(\xi_{i, 1}^{(n)}, \ldots, \xi_{i, d}^{(n)}\right)^{\prime}
$$

where $\xi_{i, k}^{(n)}, k=1,2 \ldots, d$, are independent copies of $\xi_{i}^{(n)}$ in Lemma 4. Define

$$
\eta_{n}(t)=\sum_{i=1}^{\lfloor n t\rfloor} \eta_{i}^{(n)}
$$

Then, we can get that $\left\{\eta^{(n)}\right\}_{n \in \mathbb{N}}=\left\{\eta_{i}^{(n)}, \mathscr{F}_{i}^{n}\right\}$ is still a sequence of square integrable martingale differences on the probability space $(\Omega, \mathscr{F}, \mathbb{P})$. Inspired by Lemma 4 , we have the following lemma.

Lemma 6. Under conditions (7) and (8), the sequence of processes $\eta_{n}(t)$ converges in law to a d-dimensional Brownian motion $W$, as $n \rightarrow \infty$.

Noting that $W^{i}(u), i=1, \ldots, d$, are mutually independent and so are $\xi_{k, i}^{(n)}$, we can directly get Lemma 6 from Lemma 4 and Theorem 11.4.4 in Whitt [17, Chapter 12]. 
Inspired by Lemma 6 and (5), we construct the approximation sequence by

$$
X_{n}(t)=\sum_{i=1}^{\lfloor n t\rfloor} n \int_{(i-1) / n}^{i / n}\left(\frac{\lfloor n t\rfloor}{n}-u\right)_{+}^{D-(I / 2)} \eta_{i}^{(n)} d u .
$$

Our main objective in this paper is to explain and prove the following theorem.

Theorem 7. The sequence of processes $\left\{X_{n}(t), t \in[0,1]\right\}$ given by (12), as $n \rightarrow \infty$, converges weakly to the operator fractional Brownian motion $X$ given by (5).

In the rest of this paper, most of the estimates contain unspecified constants. An unspecified positive and finite constant will be denoted by $\widetilde{K}$, which may not be the same in each occurrence.

\section{Proof of Theorem 7}

In order to prove the main result of this paper, we need a technical lemma. Before we state this technical lemma, we first introduce the following notation:

$$
\begin{gathered}
K(t, s)=(t-s)_{+}^{D-(I / 2)}=\left(K_{i, j}(t, s)\right)_{d \times d}, \\
K^{n}(t, s)=\left(\frac{\lfloor n t\rfloor}{n}-s\right)_{+}^{D-(I / 2)}=\left(K_{i, j}^{n}(t, s)\right)_{d \times d} .
\end{gathered}
$$

The technical lemma is as follows.

Lemma 8. For any $k, j \in\{1,2, \ldots, d\}$,

$$
\begin{aligned}
& \sum_{i=1}^{n} n^{2} \int_{(i-1) / n}^{i / n} K_{k, j}^{n}\left(t_{l}, s\right) d s \int_{(i-1) / n}^{i / n} K_{k, j}^{n}\left(t_{q}, s\right) d s\left(\xi_{i, j}^{(n)}\right)^{2} \\
& \longrightarrow \int_{0}^{1} K_{k, j}\left(t_{l}, s\right) K_{k, j}\left(t_{q}, s\right) d s, \quad \text { a.s. } \\
& \text { for } t_{l}, t_{q} \in[0,1] \text {, as } n \rightarrow \infty .
\end{aligned}
$$

Before we prove it, we need the following lemma which is due to Maejima and Mason [14].

Lemma 9. Let $D \in \operatorname{End}\left(\mathbb{R}^{d}\right)$. If $\lambda_{D}>0$ and $r>0$, then, for any $\delta>0$, there exist positive constants $K_{1}$ and $K_{2}$ such that

$$
\left\|r^{D}\right\| \leq \begin{cases}K_{1} r^{\lambda_{D}-\delta}, & \text { for all } r \leq 1 \\ K_{2} r^{\Lambda_{D}+\delta}, & \text { for all } r \geq 1\end{cases}
$$

Next, we give the detailed proof of Lemma 8.

Proof of Lemma 8. In order to simplify the discussion, we split the proof into two steps.

Step 1 . We claim that, for any $t \in[0,1]$,

$$
\begin{gathered}
\sum_{i=1}^{n} n^{2}\left(\int_{(i-1) / n}^{i / n} K_{k, j}(t, s) d s\right)^{2}\left(\xi_{i, j}^{(n)}\right)^{2} \\
\longrightarrow \int_{0}^{1} K_{k, j}^{2}(t, s) d s, \quad \text { a.s., }
\end{gathered}
$$

as $n \rightarrow \infty$.
For convenience, define

$$
G_{n}(t, u)=n \sum_{i=1}^{n} 1_{((i-1) / n, i / n]}(u) \int_{(i-1) / n}^{i / n} K_{k, j}(t, s) d s \frac{\xi_{i, j}^{(n)}}{(\sqrt{n})^{-1}} .
$$

Therefore, we have

$$
\begin{aligned}
\int_{0}^{1} G_{n}^{2}(t, u) d u & =\sum_{i=1}^{n} n^{2}\left(\int_{(i-1) / n}^{i / n} K_{k, j}(t, s) d s\right)^{2}\left(\xi_{i, j}^{(n)}\right)^{2} \\
& \leq \sum_{i=1}^{n} n \int_{(i-1) / n}^{i / n}\left(K_{k, j}(t, s)\right)^{2} d s\left(\xi_{i, j}^{(n)}\right)^{2}
\end{aligned}
$$

where we have used the Cauchy-Schwartz inequality and by (7).

Therefore,

$$
\int_{0}^{1} G_{n}^{2}(t, u) d u \leq \widetilde{K} \int_{0}^{1}\left(K_{k, j}(t, s)\right)^{2} d s .
$$

On the other hand, by (3) and Lemma 9,

$$
\left|K_{k, j}(t, s)\right| \leq\|K(t, s)\| \leq \widetilde{K}(t-s)_{+}^{\left(\lambda_{D^{-}}-\right)_{-(1 / 2)}},
$$

since $t-s \in[0,1]$.

By (20) and (21), we have

$$
\begin{aligned}
\int_{0}^{1} G_{n}^{2}(t, u) d u & \leq \widetilde{K} \int_{0}^{1}\left(K_{k, j}(t, s)\right)^{2} d s \\
& \leq \widetilde{K} \int_{0}^{1}(t-s)_{+}^{2\left(\lambda_{D}-\delta\right)-1}<\infty,
\end{aligned}
$$

since $\lambda_{D}-\delta>1 / 2$. Therefore, $\left\{G_{n}^{2}(t, u)\right\}$ is uniformly integrable.

On the other hand, we have, for any $u \in(0,1]$,

$$
G_{n}^{2}(t, u) \longrightarrow K_{k, j}^{2}(t, u), \quad \text { a.s., }
$$

since, for $u \in((i-1) / n, i / n]$,

$$
\left(n \int_{(i-1) / n}^{i / n} K_{k, j}(t, s) d s\right)^{2} \longrightarrow K_{k, j}^{2}(t, u), \quad \text { as } n \longrightarrow \infty,
$$

and condition (6).

By (22) and (23), we get that as $n \rightarrow \infty$

$$
\int_{0}^{1} G_{n}^{2}(t, u) d u \longrightarrow \int_{0}^{1} K_{k, j}^{2}(t, s) d s, \quad \text { a.s. }
$$

Therefore, (17) holds.

Step 2. We prove the original claim. In order to simplify the discussion, we let $t_{q}^{n}=\left\lfloor n t_{q}\right\rfloor / n$ and $t_{l}^{n}=\left\lfloor n t_{l}\right\rfloor / n$. By (17), we can get that, for $t_{l}, t_{q} \in[0,1]$,

$$
\begin{gathered}
\sum_{i=1}^{n} n^{2} \int_{(i-1) / n}^{i / n} K_{k, j}\left(t_{l}, s\right) d s \int_{(i-1) / n}^{i / n} K_{k, j}\left(t_{q}, s\right) d s\left(\xi_{i, j}^{(n)}\right)^{2} \\
\longrightarrow \int_{0}^{1} K_{k, j}\left(t_{l}, s\right) K_{k, j}\left(t_{q}, s\right) d s, \quad \text { a.s. }
\end{gathered}
$$


as $n \rightarrow \infty$. In fact, it follows from (17) that

$$
\begin{gathered}
\sum_{i=1}^{n} n^{2}\left(\int_{(i-1) / n}^{i / n} K_{k, j}\left(t_{l}, s\right)+K_{k, j}\left(t_{q}, s\right) d s\right)^{2}\left(\xi_{i, j}^{(n)}\right)^{2} \\
\longrightarrow \int_{0}^{1}\left(K_{k, j}\left(t_{l}, s\right)+K_{k, j}\left(t_{q}, s\right)\right)^{2} d s .
\end{gathered}
$$

On the other hand, we have

$$
\begin{aligned}
& \left(\int_{(i-1) / n}^{i / n} K_{k, j}\left(t_{l}, s\right)+K_{k, j}\left(t_{q}, s\right) d s\right)^{2} \\
& \quad=\left(\int_{(i-1) / n}^{i / n} K_{k, j}\left(t_{l}, s\right) d s\right)^{2}+\left(\int_{(i-1) / n}^{i / n} K_{k, j}\left(t_{q}, s\right) d s\right)^{2} \\
& \quad+2 \int_{(i-1) / n}^{i / n} K_{k, j}\left(t_{l}, s\right) d s \int_{(i-1) / n}^{i / n} K_{k, j}\left(t_{q}, s\right) d s .
\end{aligned}
$$

Hence (17), (27), and (28) imply (26).

Therefore, in order to prove (15), it suffices to prove that

$$
\begin{aligned}
& \sum_{i=1}^{n} n^{2}\left(\int_{(i-1) / n}^{i / n} K_{k, j}\left(t_{l}, s\right) d s \int_{(i-1) / n}^{i / n} K_{k, j}\left(t_{q}, s\right) d s\right. \\
&\left.-\int_{(i-1) / n}^{i / n} K_{k, j}\left(t_{l}^{n}, s\right) d s \int_{(i-1) / n}^{i / n} K_{k, j}\left(t_{q}^{n}, s\right) d s\right)\left(\xi_{i, j}^{(n)}\right)^{2} \\
& \longrightarrow 0, \quad \text { a.s. }
\end{aligned}
$$

as $n \rightarrow \infty$.

For the left-hand side of (29), we have

$$
\begin{aligned}
\int_{(i-1) / n}^{i / n} & K_{k, j}\left(t_{l}, s\right) d s \int_{(i-1) / n}^{i / n} K_{k, j}\left(t_{q}, s\right) d s \\
& -\int_{(i-1) / n}^{i / n} K_{k, j}\left(t_{l}^{n}, s\right) d s \int_{(i-1) / n}^{i / n} K_{k, j}\left(t_{q}^{n}, s\right) d s \\
= & \int_{(i-1) / n}^{i / n} K_{k, j}\left(t_{l}, s\right) d s \int_{(i-1) / n}^{i / n}\left(K_{k, j}\left(t_{q}, s\right)\right. \\
& -\int_{(i-1) / n}^{i / n}\left(K_{k, j}\left(t_{l}^{n}, s\right)-K_{k, j}\left(t_{l}, s\right)\right) d s \\
& \left.\times \int_{(i-1) / n}^{i / n}\left(K_{k, j}\left(t_{q}^{n}, s\right)-K_{k, j}^{n}, s\right)\right) d s \\
& \left.+\int_{(i-1) / n}^{i / n} K_{k, j}\left(t_{q}, s\right)\right) d s
\end{aligned}
$$

By (3), we have

$$
\left|K_{k, j}\left(t_{q}, s\right)-K_{k, j}\left(t_{q}^{n}, s\right)\right| \leq\left\|K\left(t_{q}, s\right)-K\left(t_{q}^{n}, s\right)\right\| .
$$

On the other hand, using the same method as in the proof of inequality (76) below, we have

$$
\begin{aligned}
& \sum_{i=1}^{n} \int_{(i-1) / n}^{i / n}\left\|K\left(t_{q}, s\right)-K\left(t_{q}^{n}, s\right)\right\| d s \\
& \quad \leq \int_{0}^{1}\left\|K\left(t_{q}, s\right)-K\left(t_{q}^{n}, s\right)\right\| d s \leq \widetilde{K}\left(t_{q}^{n}-t_{q}\right)^{2 H},
\end{aligned}
$$

where $H=\lambda_{D}-\delta$.

By (7) and (30), (29) can be bounded by

$$
\begin{aligned}
& \widetilde{K} n \int_{0}^{1}\left\|K\left(t_{l}, s\right)\right\| d s \int_{0}^{1}\left\|K\left(t_{q}, s\right)-K\left(t_{q}^{n}, s\right)\right\| d s \\
& \quad+\widetilde{K} n \int_{0}^{1}\left\|K\left(t_{l}^{n}, s\right)-K\left(t_{l}, s\right)\right\| d s \\
& \quad \times \int_{0}^{1}\left\|K\left(t_{q}^{n}, s\right)-K\left(t_{q}, s\right)\right\| d s \\
& \quad+\widetilde{K} n \int_{0}^{1}\left\|K\left(t_{q}, s\right)\right\| d s \int_{0}^{1}\left\|K\left(t_{l}, s\right)-K\left(t_{l}^{n}, s\right)\right\| d s .
\end{aligned}
$$

It follows from (22), (32), and (33) that the left-hand side of (29) can be bounded by

$$
\widetilde{K} n^{1-2 H},
$$

since $\left|t_{q}^{n}-t_{q}\right| \leq 1 / n$ and $\left|t_{l}^{n}-t_{l}\right| \leq 1 / n$.

From (29) and (34), we can easily prove the lemma.

From the proof of Lemma 8 and (3), we can easily get the following.

Corollary 10. Let $H(t, s)=\sum_{k=1}^{d} a_{k} K_{k, j}^{n}\left(t_{l}, s\right)$ for any $a_{k} \in \mathbb{R}$. Then

$$
\begin{gathered}
\sum_{i=1}^{n} n^{2} \int_{(i-1) / n}^{i / n} H\left(t_{l}, s\right) d s \int_{(i-1) / n}^{i / n} H\left(t_{q}, s\right) d s\left(\xi_{i, j}^{(n)}\right)^{2} \\
\longrightarrow \int_{0}^{1} H\left(t_{l}, s\right) H\left(t_{q}, s\right) d s, \quad \text { a.s. }
\end{gathered}
$$

for any $t_{l}, t_{q} \in(0,1]$.

Next, we prove the main result of this paper. Before we give the details, we first introduce a technical tool.

Lemma 11. Let $t \in(0,1], \sigma_{t}^{2}>0$, and let $\left\{\xi^{(n)}\right\}$ be a sequence of martingale differences as in Section 2 and satisfy the following Lindberg condition: for $\epsilon>0$

$$
\sum_{i=1}^{\lfloor n t\rfloor} \mathbb{E}\left[\left(\xi_{i}^{(n)}\right)^{2} I_{\left\{\left|\xi_{i}^{(n)}\right|>\epsilon\right\}} \mid \mathscr{F}_{i-1}^{n}\right] \stackrel{P}{\longrightarrow} 0 .
$$

Then

$$
\sum_{i=1}^{\lfloor n t\rfloor}\left(\xi_{i}^{(n)}\right)^{2} \stackrel{P}{\longrightarrow} \sigma_{t}^{2}
$$


implies

$$
B^{n}(t) \stackrel{D}{\longrightarrow} \mathcal{N} \sim N\left(0, \sigma_{t}^{2}\right)
$$

where $\stackrel{D}{\rightarrow}$ denotes convergence in distribution.

Lemma 11 can be found in Shiryaev [18, page 511].

Proof of Theorem 7. We will prove this theorem by two steps.

Step 1. First, we show that the finite-dimensional distributions of $X_{n}$ converge to those of $X$. It suffices to prove that, for any $q \in \mathbb{N}, a_{1}, \ldots, a_{q} \in \mathbb{R}$, and $t_{1}, \ldots, t_{q} \in[0,1]$,

$$
\sum_{l=1}^{q} a_{l} X_{n}\left(t_{l}\right) \stackrel{D}{\longrightarrow} \sum_{l=1}^{q} a_{l} X\left(t_{l}\right)
$$

By the Cramér-Wold device (see Whitt [17, Chapter 4]), in order to prove (39), we only need to show

$$
\sum_{l=1}^{q} a_{l} b X_{n}\left(t_{l}\right) \stackrel{D}{\longrightarrow} \sum_{l=1}^{q} a_{l} b X\left(t_{l}\right)
$$

for any vector $b=\left(b^{(1)}, \ldots, b^{(d)}\right) \in \mathbb{R}^{d}$.

For convenience, define

$$
X_{n}(t)=\left(X_{1}^{(n)}(t), \ldots, X_{d}^{(n)}(t)\right)^{\prime},
$$

where

$$
X_{j}^{(n)}(t)=n \sum_{i=1}^{\lfloor n t\rfloor} \int_{(i-1) / n}^{i / n} K_{j}^{n}(t, s) \eta_{i}^{(n)} d s
$$

with

$$
\begin{aligned}
K_{j}^{n}(t, s) & =\left(K_{j, 1}^{n}(t, s), \ldots, K_{j, d}^{n}(t, s)\right), \\
X(t) & =\left(X^{(1)}(t), \ldots, X^{(d)}(t)\right)^{\prime},
\end{aligned}
$$

where

$$
X^{(j)}(t)=\int_{0}^{t} K_{j}(t, s) d W(s)
$$

with

$$
K_{j}(t, s)=\left(K_{j, 1}(t, s), \ldots, K_{j, d}(t, s)\right)
$$

By some calculations, we can get that (40) is equivalent to

$$
\begin{gathered}
\sum_{l=1}^{q} \sum_{k=1}^{d} \sum_{j=1}^{d} \sum_{i=1}^{\left\lfloor n t_{l}\right\rfloor} n \int_{(i-1) / n}^{i / n} a_{l} b^{(k)} K_{k, j}^{n}\left(t_{l}, s\right) \xi_{i, j}^{(n)} d s \\
\stackrel{D}{\longrightarrow} \sum_{l=1}^{q} \sum_{k=1}^{d} \sum_{j=1}^{d} \int_{0}^{t} a_{l} b^{(k)} K_{k, j}\left(t_{l}, s\right) d W^{j}(s) .
\end{gathered}
$$

In order to simplify the discussion, we define

$$
\begin{gathered}
\bar{X}^{n}(l, k, j)=\sum_{i=1}^{\left\lfloor n t_{l}\right\rfloor} n \int_{(i-1) / n}^{i / n} K_{k, j}^{n}\left(t_{l}, s\right) \xi_{i, j}^{(n)} d s, \\
\bar{X}(l, k, j)=\int_{0}^{t_{l}} K_{k, j}\left(t_{l}, s\right) d W^{j}(s) .
\end{gathered}
$$

Hence, (46) can be rewritten as follows:

$$
\sum_{l=1}^{q} \sum_{k, j=1}^{d} a_{l} b^{(k)} \bar{X}^{n}(l, k, j) \stackrel{D}{\longrightarrow} \sum_{l=1}^{q} \sum_{k, j=1}^{d} a_{l} b^{(k)} \bar{X}(l, k, j) .
$$

By the independence of $\xi_{i, j}^{(n)}, j=1, \ldots, d$, it suffices to show that for every $j \in\{1, \ldots, d\}$

$$
\sum_{l=1}^{q} \sum_{k=1}^{d} a_{l} b^{(k)} \bar{X}^{n}(l, k, j) \stackrel{D}{\longrightarrow} \sum_{l=1}^{q} \sum_{k=1}^{d} a_{l} b^{(k)} \bar{X}(l, k, j) .
$$

We will prove (49) by Lemma 11 . We first prove that the Lindeberg condition holds in our case. For convenience, define

$$
Z_{k, i}^{n}(t)=n \int_{(i-1) / n}^{i / n} K_{k, j}^{n}(t, s) \xi_{i, j}^{(n)} d s
$$

We have

$$
\begin{aligned}
\left(Z_{k, i}^{n}(t)\right)^{2} & =n^{2}\left(\xi_{i, j}^{(n)}\right)^{2}\left(\int_{(i-1) / n}^{i / n} K_{k, j}^{n}(t, s) d s\right)^{2} \\
& \leq n\left(\xi_{i, j}^{(n)}\right)^{2} \int_{(i-1) / n}^{i / n}\left(K_{k, j}^{n}(t, s)\right)^{2} d s
\end{aligned}
$$

where we have used the Hölder inequality. By (3), we have

$$
\left|K_{k, j}^{n}(t, s)\right| \leq\left\|(t-s)_{+}^{D-(I / 2)}\right\| .
$$

By Lemma 9, we have

$$
\left\|(t-s)_{+}^{D-(I / 2)}\right\| \leq \widetilde{K}(t-s)_{+}^{\lambda_{D^{-}}(1 / 2)-\delta},
$$

since $t, s \in[0,1]$.

By (52) and (53),

$$
\begin{aligned}
& \int_{(i-1) / n}^{i / n}\left\|K_{k, j}(t, s)\right\|^{2} d s \leq \widetilde{K} \int_{(i-1) / n}^{i / n}(t-s)_{+}^{2\left(\lambda_{D}-\delta\right)-1} d s \\
& \quad \leq \widetilde{K} \int_{0}^{1 / n}(1-s)^{2\left(\lambda_{D}-\delta\right)-1} d s,
\end{aligned}
$$

since $(1-s)^{2\left(\lambda_{D}-\delta\right)-1}$ with $\lambda_{D}-\delta>1 / 2$ is decreasing in $s$. It follows from (51) and (54) that

$$
\left(Z_{k, i}^{n}(t)\right)^{2} \leq \widetilde{K} n\left(\xi_{i, j}^{(n)}\right)^{2} \delta_{n}
$$

with $\delta_{n}=\int_{0}^{1 / n}(1-s)^{2\left(\lambda_{D}-\delta\right)-1} d s$. 
On the other hand, from (14), we get, for any $s \geq\lfloor n t\rfloor / n$,

$$
K_{k, j}^{n}(t, s)=0 .
$$

Hence, by (56),

$$
\sum_{l=1}^{q} \sum_{k=1}^{d} a_{l} b^{(k)} \bar{X}^{n}(l, k, j)=\sum_{i=1}^{n} \sum_{l=1}^{q} \sum_{k=1}^{d} a_{l} b^{(k)} Z_{k, i}^{n}\left(t_{l}\right) .
$$

Finally, we have

$$
\left(\sum_{l=1}^{q} \sum_{k=1}^{d} a_{l} b^{(k)} Z_{k, i}^{n}\left(t_{l}\right)\right)^{2} \leq \widetilde{K} \sum_{l=1}^{q} \sum_{k=1}^{d}\left(b^{(k)}\right)^{2} a_{l}^{2}\left(Z_{k, i}^{n}\left(t_{l}\right)\right)^{2} .
$$

Combining (54) and (58), we have

$$
\left(\sum_{l=1}^{q} \sum_{k=1}^{d} a_{l} b^{(k)} Z_{k, i}^{n}\left(t_{l}\right)\right)^{2} \leq \widetilde{K} n\left(\xi_{i, j}^{n}\right)^{2} \delta_{n}
$$

Noting that

$$
\begin{aligned}
& \left\{\left|\sum_{l=1}^{q} \sum_{k=1}^{d} a_{l} b^{(k)} Z_{k, i}^{n}\left(t_{l}\right)\right|>\epsilon\right\} \\
& =\left\{\left(\sum_{l=1}^{q} \sum_{k=1}^{d} a_{l} b^{(k)} Z_{k, i}^{n}\left(t_{l}\right)\right)^{2}>\epsilon^{2}\right\},
\end{aligned}
$$

from (59), we have

$$
\left\{\left|\sum_{l=1}^{q} \sum_{k=1}^{d} a_{l} b^{(k)} Z_{k, i}^{n}\left(t_{l}\right)\right|>\epsilon\right\} \subset\left\{\widetilde{K} n\left(\xi_{i, k}^{n}\right)^{2} \delta_{n}>\epsilon^{2}\right\} .
$$

Therefore, by (59) and (61),

$$
\begin{aligned}
& \mathbb{E}\left(\left(\sum_{l=1}^{q} \sum_{k=1}^{d} a_{l} b^{(k)} Z_{k, i}^{n}\left(t_{l}\right)\right)^{2} I_{\left\{\left|\sum_{l=1}^{q} \sum_{k=1}^{d} a_{l} b^{(k)} Z_{k, i}^{n}\left(t_{l}\right)\right|>\xi\right\}} \mid \mathscr{F}_{i-1}^{n}\right) \\
& \leq \widetilde{K} n\left(\xi_{i, j}^{n}\right)^{2} \delta_{n} \mathbb{E}\left(I_{\left\{\widetilde{K} n\left(\xi_{i, j}^{n}\right)^{2} \delta_{n}>\epsilon^{2}\right\}} \mid \mathscr{F}_{i-1}^{n}\right) \\
& \leq \widetilde{K} \delta_{n} \mathbb{E}\left(I_{\left\{\widetilde{K} \delta_{n}>\epsilon^{2}\right\}} \mid \mathscr{F}_{i-1}^{n}\right) .
\end{aligned}
$$

Combining (57) and (62), one can easily prove that, as $n$ approaches $\infty$,

$$
\sum_{i=1}^{n} \mathbb{E}\left(\left(\sum_{l=1}^{q} \sum_{k=1}^{d} a_{l} b^{(k)} Z_{k, i}^{n}\right)^{2} I_{\left\{\left|\sum_{l=1}^{q} \sum_{k=1}^{d} a_{l} b^{(k)} Z_{k, i}^{n}\right|>\xi\right\}} \mid \mathscr{F}_{i-1}^{n}\right) \longrightarrow 0 .
$$

Hence, the Lindeberg condition holds.

Next, we show that condition (37) holds. We first study the right-hand side of (49). We have

$$
\sum_{l=1}^{q} \sum_{k=1}^{d} a_{l} b^{(k)} \bar{X}(l, k, j)=\sum_{l=1}^{q} a_{l} \widetilde{W}\left(t_{l}\right)
$$

where

$$
\widetilde{W}(t)=\int_{0}^{t}\left[\sum_{k=1}^{d} b^{(k)} K_{k, j}(t, s)\right] d W^{j}(s)=\int_{0}^{t} \bar{K}(t, s) d W^{j}(s),
$$

with

$$
\bar{K}(t, s)=\sum_{k=1}^{d} b^{(k)} K_{k, j}(t, s) .
$$

Combining (64) and (65), we have

$$
\begin{aligned}
& \mathbb{E}\left[\sum_{l=1}^{q} \sum_{k=1}^{d} a_{l} b^{(k)} \bar{X}(l, k, j)\right]^{2} \\
& =\mathbb{E}\left[\sum_{l=1}^{q} a_{l} \widetilde{W}\left(t_{l}\right)\right]^{2} \\
& =\sum_{l, j=1}^{q} a_{j} a_{l} \int_{0}^{1} \bar{K}\left(t_{j}, s\right) \bar{K}\left(t_{l}, s\right) d s .
\end{aligned}
$$

Hence, in order to show condition (37), we only need to show

$$
\begin{aligned}
& \sum_{i=1}^{n}\left(\sum_{l=1}^{q} \sum_{k=1}^{d} a_{l} b^{(k)} Z_{k, i}^{n}\right)^{2} \\
& \quad \stackrel{P}{\longrightarrow} \sum_{l, j=1}^{q} a_{j} a_{l} \int_{0}^{1} \bar{K}\left(t_{j}, s\right) \bar{K}\left(t_{l}, s\right) d s .
\end{aligned}
$$

Now, we focus on the left-hand side of (68). Similar to (64), we have

$$
\sum_{l=1}^{q} \sum_{k=1}^{d} a_{l} b^{(k)} Z_{k, i}^{n}=\sum_{l=1}^{q} a_{l} \bar{Z}_{l, i}^{n}
$$

where

$$
\bar{Z}_{l, i}^{n}=n \int_{(i-1) / n}^{i / n} \bar{K}_{j}^{n}\left(t_{l}, s\right) \xi_{i, j}^{(n)} d s,
$$

with $\bar{K}_{j}^{n}\left(t_{l}, s\right)=\sum_{k=1}^{d} b^{(k)} K_{k, j}^{n}\left(t_{l}, s\right)$. Hence,

$$
\begin{aligned}
& \sum_{i=1}^{n}\left(\sum_{l=1}^{q} \sum_{k=1}^{d} a_{l} b^{(k)} Z_{k, i}^{n}\right)^{2} \\
& =\sum_{i=1}^{n} \sum_{l_{1}, l_{2}=1}^{q} n^{2} a_{l_{1}} a_{l_{2}} \int_{(i-1) / n}^{i / n} \bar{K}_{j}\left(t_{l_{1}}, s\right) d s \\
& \quad \times \int_{(i-1) / n}^{i / n} \bar{K}_{j}\left(t_{l_{2}}, s\right) d s\left(\xi_{i, j}^{(n)}\right)^{2} .
\end{aligned}
$$

It follows from Corollary 10 that the right-hand side of (71) converges to

$$
\sum_{l_{1}, l_{2}=1}^{q} a_{l_{1}} a_{l_{2}} \int_{0}^{1} \bar{K}_{j}\left(t_{l_{1}}, s\right) \bar{K}_{j}\left(t_{l_{2}}, s\right) d s, \quad \text { a.s. }
$$


as $n \rightarrow \infty$. On the other hand, one can easily get that

$$
\mathbb{E}\left[\widetilde{W}\left(t_{l}\right) \widetilde{W}\left(t_{k}\right)\right]=\int_{0}^{1} \bar{K}\left(t_{l}, s\right) \bar{K}\left(t_{k}, s\right) d s .
$$

By (67), (72), and (73), we get condition (37).

Step 2. We need to prove the tightness of the sequence $\left\{X_{n}(t)\right\}$.

By some calculations,

$$
\begin{aligned}
& \mathbb{E}\left(\left\|X_{n}(t)-X_{n}(s)\right\|^{2}\right) \\
& \quad \leq \widetilde{K} \int_{0}^{1}\left\|\left(\frac{\lfloor n t\rfloor}{n}-u\right)_{+}^{D-(I / 2)}-\left(\frac{\lfloor n s\rfloor}{n}-u\right)_{+}^{D-(I / 2)}\right\|^{2} d u .
\end{aligned}
$$

In order to simplify the discussion, let

$$
\tilde{t}=\frac{\lfloor n t\rfloor}{n}, \quad \widetilde{s}=\frac{\lfloor n s\rfloor}{n} .
$$

Next, we show that

$$
\int_{0}^{1}\left\|(\tilde{t}-u)_{+}^{D-(I / 2)}-(\widetilde{s}-u)_{+}^{D-(I / 2)}\right\|^{2} d u \leq K(\widetilde{t}-\widetilde{s})^{2 H}
$$

where $H=\lambda_{D}-\delta$.

In fact,

$$
\begin{gathered}
\int_{0}^{1}\left\|(\tilde{t}-u)_{+}^{D-(I / 2)}-(\widetilde{s}-u)_{+}^{D-(I / 2)}\right\|^{2} d u \\
=\int_{0}^{\widetilde{s}}\left\|(\widetilde{t}-u)^{D-(I / 2)}-(\widetilde{s}-u)^{D-(I / 2)}\right\|^{2} d u \\
+\int_{\widetilde{s}}^{\widetilde{t}}\left\|(\widetilde{t}-u)^{D-(I / 2)}\right\|^{2} d u .
\end{gathered}
$$

It follows from (2) and Lemma 9 that

$$
\left\|(\widetilde{t}-u)^{D-(I / 2)}\right\| \leq \widetilde{K}(\widetilde{t}-u)^{\lambda_{D^{-}-\delta-(1 / 2)}},
$$

since $u \leq \tilde{t} \in[0,1]$.

Therefore,

$$
\begin{aligned}
\int_{\widetilde{s}}^{\widetilde{t}}\left\|(\tilde{t}-u)_{+}^{D-(I / 2)}\right\|^{2} d u & \leq \widetilde{K} \int_{\widetilde{s}}^{\widetilde{t}}(\tilde{t}-u)^{2\left(\lambda_{D}-\delta\right)-1} d u \\
& =\frac{\widetilde{K}(\widetilde{t}-\widetilde{s})^{2\left(\lambda_{D}-\delta\right)}}{2\left(\lambda_{D}-\delta\right)} .
\end{aligned}
$$

Next, we deal with the first term on the right-hand side of (77). Note that

$$
\begin{aligned}
\int_{0}^{\widetilde{s}} & \left\|(\widetilde{t}-u)^{D-(I / 2)}-(\widetilde{s}-u)^{D-(I / 2)}\right\|^{2} d u \\
= & \int_{0}^{\widetilde{s}}\left\|(\tilde{t}-\widetilde{s}+u)^{D-(I / 2)}-u^{D-(I / 2)}\right\|^{2} d u \\
= & \int_{0}^{\widetilde{s} /(\widetilde{t}-\widetilde{s})} \|[(\widetilde{t}-\widetilde{s})(1+u)]^{D-(I / 2)} \\
\leq & \left\|(\widetilde{t}-\widetilde{s})^{D-(I / 2)}\right\|^{2}(\widetilde{t}-\widetilde{s}) \\
& \times \int_{0}^{\widetilde{s} /(\widetilde{t}-\widetilde{s})}\left\|(1+u)^{D-(I / 2)}-u^{D-(I / 2)}\right\|^{2} d u \\
\leq & \left\|(\widetilde{t}-\widetilde{s})^{D-(I / 2)}\right\|^{2}(\tilde{t}-\widetilde{s}) \\
& \times \int_{\mathbb{R}_{+}}\left\|(1+u)^{D-(I / 2)}-u^{D-(I / 2)}\right\|^{2} d u,
\end{aligned}
$$

where we used the fact that $(\widetilde{t} \widetilde{s})^{A}=\widetilde{t}^{A} \cdot \widetilde{s}^{A}$.

It follows from Lemma 9 and (2) that

$$
\left\|(\widetilde{t}-\widetilde{s})^{D-(I / 2)}\right\|^{2}(\widetilde{t}-\widetilde{s}) \leq \widetilde{K}(\widetilde{t}-\widetilde{s})^{2\left(\lambda_{D}-\delta\right)} .
$$

In order to prove our result, it suffices to show that

$$
\int_{\mathbb{R}_{+}}\left\|(1+u)^{D-(I / 2)}-u^{D-(I / 2)}\right\|^{2} d u<\infty .
$$

Then, in order to prove (82), it suffices to show that

$$
\int_{u \leq 1}\left\|u^{D-(I / 2)}\right\|^{2} d u<\infty
$$

and, for large enough $T>1$, that

$$
\int_{u \geq T}\left\|(1+u)^{D-(I / 2)}-u^{D-(I / 2)}\right\|^{2} d u<\infty .
$$

It follows from Lemma 9 and (2) that

$$
\left\|u^{D-(I / 2)}\right\|^{2} \leq \widetilde{K} u^{2\left(\lambda_{D}-\delta\right)-1} \quad \text { for } u \leq 1 .
$$

Hence, one can easily see that (83) holds.

Next, we show that (84) holds. We see that

$$
(1+u)^{D-(I / 2)}-u^{D-(I / 2)}=\int_{u}^{1+u}\left(D-\frac{I}{2}\right) s^{D-(I / 2)} s^{-1} d s .
$$

Then

$$
\begin{aligned}
& \left\|(1+u)^{D-(I / 2)}-u^{D-(I / 2)}\right\| \\
& \quad \leq\left\|\left(D-\frac{I}{2}\right)\right\| \int_{u}^{1+u}\left\|s^{D-(I / 2)}\right\| s^{-1} d s .
\end{aligned}
$$


It follows from Lemma 4 and (2) that

$$
\int_{u}^{1+u}\left\|s^{D-(I / 2)}\right\| s^{-1} d s \leq \int_{u}^{1+u} \widetilde{K} s^{\Lambda_{D}+\delta-(3 / 2)} d s,
$$

since $u \geq 1$.

By (87) and (88),

$$
\left\|(1+u)^{D-(I / 2)}-(u)^{D-(I / 2)}\right\|^{2} \leq \widetilde{K} u^{2\left(\Lambda_{D}+\delta\right)-3} .
$$

By (89), we have that (84) holds, since $\Lambda_{D}+\delta<1$.

Therefore, we have

$$
\mathbb{E}\left(\left\|X_{n}(t)-X_{n}(s)\right\|^{2}\right) \leq \widetilde{K}(\widetilde{t}-\widetilde{s})^{2 H} .
$$

Hence, for any $s \leq t \leq u \in[0,1]$, we have

$$
\begin{aligned}
& \mathbb{E}\left[\left\|X_{n}(t)-X_{n}(s)\right\|\left\|X_{n}(t)-X_{n}(u)\right\|\right] \\
& \leq\left[\mathbb{E}\left\|X_{n}(t)-X_{n}(s)\right\|^{2}\right]^{1 / 2}\left[\mathbb{E}\left\|X_{n}(t)-X_{n}(u)\right\|^{2}\right]^{1 / 2} \\
& \quad \leq \widetilde{K}\left|\frac{\lfloor n t\rfloor}{n}-\frac{\lfloor n s\rfloor}{n}\right|^{H}\left|\frac{\lfloor n u\rfloor}{n}-\frac{\lfloor n t\rfloor}{n}\right|^{H} \\
& \quad \leq \widetilde{K}\left|\frac{\lfloor n u\rfloor}{n}-\frac{\lfloor n s\rfloor}{n}\right|^{2 H} .
\end{aligned}
$$

If $u-s \geq 1 / n$, then one can easily see that

$$
\mathbb{E}\left[\left\|X_{n}(t)-X_{n}(s)\right\|\left\|X_{n}(t)-X_{n}(u)\right\|\right] \leq \widetilde{K}(u-s)^{2 H} .
$$

On the other hand, if $u-s<1 / n$, then either $s$ and $t$ or $t$ and $u$ belong to the interval $[i / n,(i+1) / n]$ for some $i$. Thus, the left-hand side of (91) is zero. Therefore, (92) still holds for this case. Hence, it follows from Ethier and Kurtz [19, Chapter 3] that $\left\{X_{n}(t)\right\}$ is tight, since $\lambda_{D}-\delta>1 / 2$.

By Theorem 7.8 in Ethier and Kurtz [19, Chapter 3], we get that Theorem 7 holds. This completes the proof.

\section{Conflict of Interests}

The authors declare that there is no conflict of interests regarding the publication of this paper.

\section{Acknowledgments}

The authors would like to thank the two referees for their helpful suggestions and comments which have improved the paper. This work was supported by the National Natural Science Foundation of China (no. 11361007), the Guangxi Natural Science Foundation (nos. 2012GXNSFBA053010 and 2014GXNSFCA118001), and the National Science Council of Taiwan (NSC 101-2118-M- 007-001-MY2).

\section{References}

[1] N. Enriquez, "A simple construction of the fractional Brownian motion," Stochastic Processes and their Applications, vol. 109, no. 2, pp. 203-223, 2004.
[2] A. Nieminen, "Fractional Brownian motion and martingaledifferences," Statistics \& Probability Letters, vol. 70, no. 1, pp. 110, 2004.

[3] T. Sottinen, "Fractional Brownian motion, random walks and binary market models," Finance and Stochastics, vol. 5, no. 3, pp. 343-355, 2001.

[4] Y. Li and H. Dai, "Approximations of fractional Brownian motion," Bernoulli, vol. 17, no. 4, pp. 1195-1216, 2011.

[5] C.-F. Chung, "Sample means, sample autocovariances, and linear regression of stationary multivariate long memory processes," Econometric Theory, vol. 18, no. 1, pp. 51-78, 2002.

[6] J. Davidson and R. M. de Jong, "The functional central limit theorem and weak convergence to stochastic integrals. II. Fractionally integrated processes," Econometric Theory, vol. 16, no. 5, pp. 643-666, 2000.

[7] J. J. Dolado and F. Marmol, "Asymptotic inference results for multivariate long-memory processes," The Econometrics Journal, vol. 7, no. 1, pp. 168-190, 2004.

[8] P. M. Robinson, "Multiple local Whittle estimation in stationary systems," The Annals of Statistics, vol. 36, no. 5, pp. 2508-2530, 2008.

[9] D. Marinucci and P. Robinson, "Weak convergence of multivariate fractional processes," Stochastic Processes and Their Applications, vol. 86, no. 1, pp. 103-120, 2000.

[10] R. Delgado, "A reflected fBm limit for fluid models with ON/OFF sources under heavy traffic," Stochastic Processes and Their Applications, vol. 117, no. 2, pp. 188-201, 2007.

[11] T. Konstantopoulos and S. J. Lin, "Fractional Brownian approximations of queuing networks," in Stochastic Networks, vol. 117 of Lecture Notes in Statistics, pp. 257-273, Springer, New York, NY, USA, 1996.

[12] H. Dai, "Convergence in law to operator fractional Brownian motions," Journal of Theoretical Probability, vol. 26, no. 3, pp. 676-696, 2013.

[13] H. Dai, "Convergence in law to operator fractional Brownian motion of Riemann-Liouville type," Acta Mathematica Sinica, English Series, vol. 29, pp. 777-788, 2013.

[14] M. Maejima and J. D. Mason, "Operator-self-similar stable processes," Stochastic Processes and Their Applications, vol. 54, no. 1, pp. 139-163, 1994.

[15] P. Lévy, Random Functions: General Theory with Special Reference to Laplacian Random Functions, University of California Press, Oakland, Calif, USA, 1953.

[16] J. Jacod and A. N. Shiryaev, Limit Theorems for Stochastic Processes, vol. 288 of Fundamental Principles of Mathematical Sciences, Springer, Berlin, Germany, 1987.

[17] W. Whitt, Stochastic-Process Limits, Springer, New York, NY, USA, 2002.

[18] A. N. Shiryayev, Probability, Springer, New York, NY, USA, 1984.

[19] S. N. Ethier and T. G. Kurtz, Markov Processes: Characterization and Convergence, Wiley Series in Probability and Mathematical Statistics: Probability and Mathematical Statistics, John Wiley \& Sons, Hoboken, NJ, USA, 1986. 


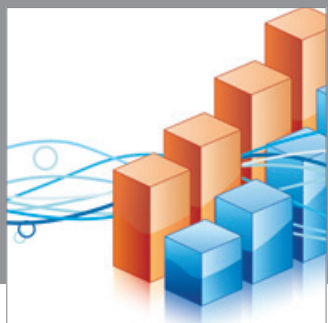

Advances in

Operations Research

mansans

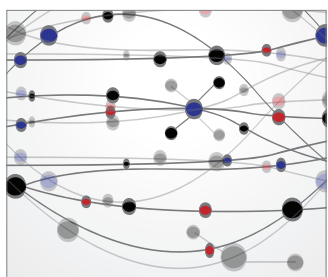

The Scientific World Journal
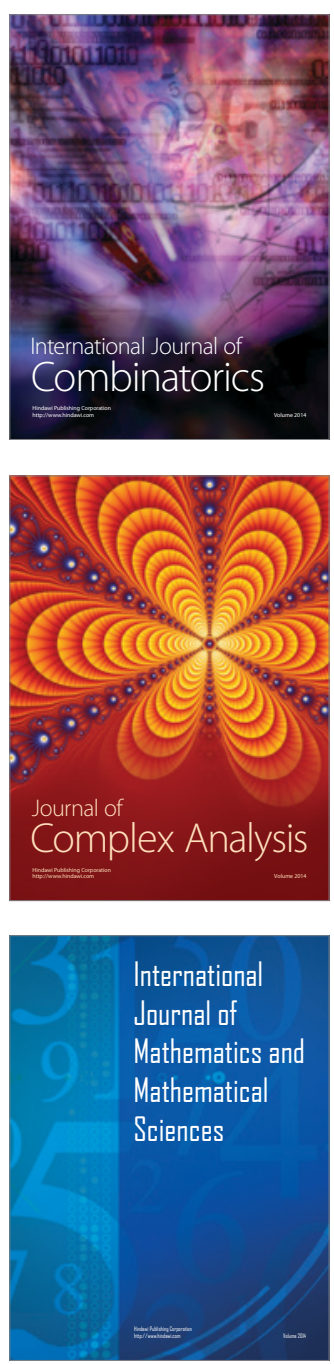
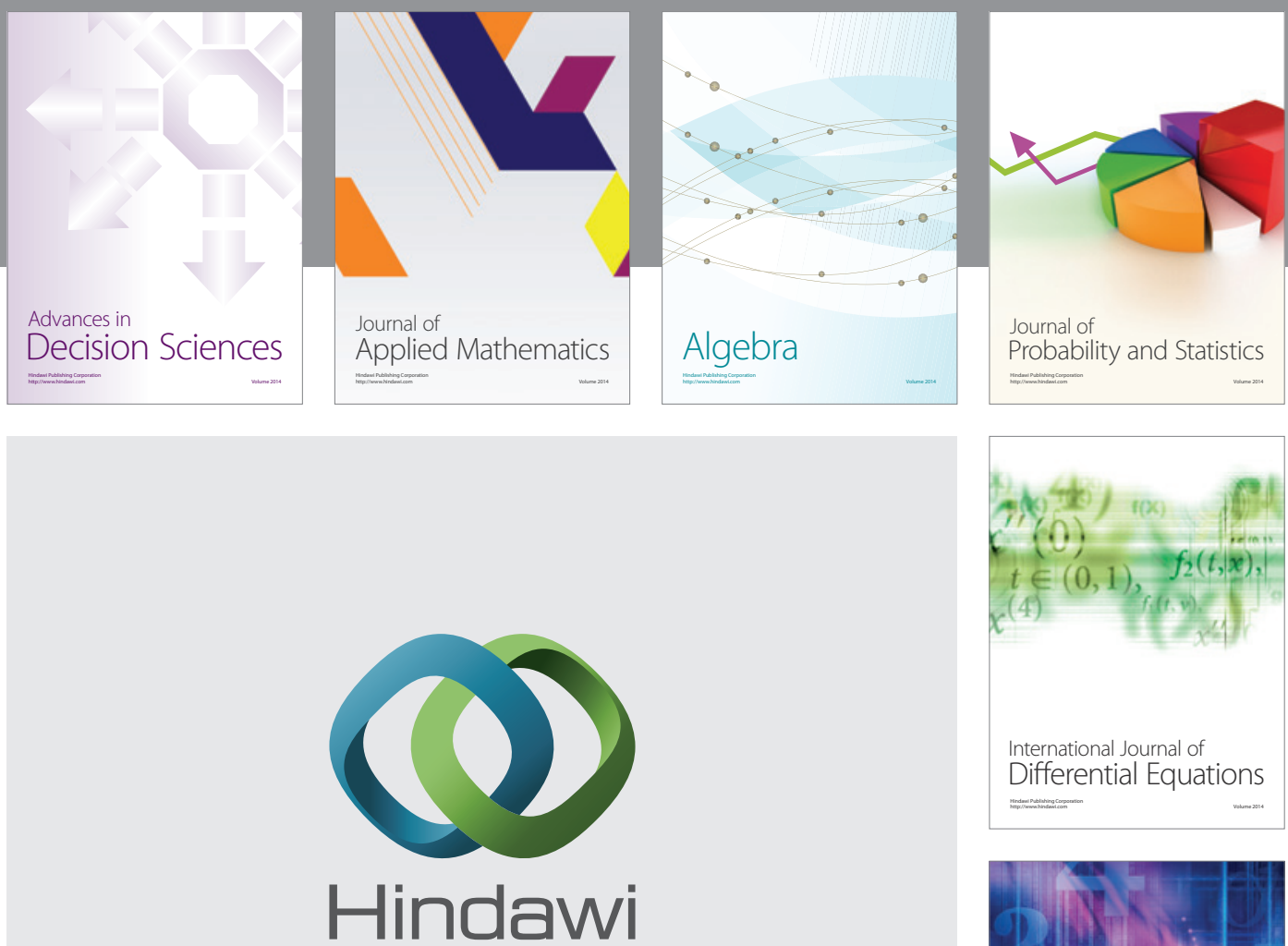

Submit your manuscripts at http://www.hindawi.com
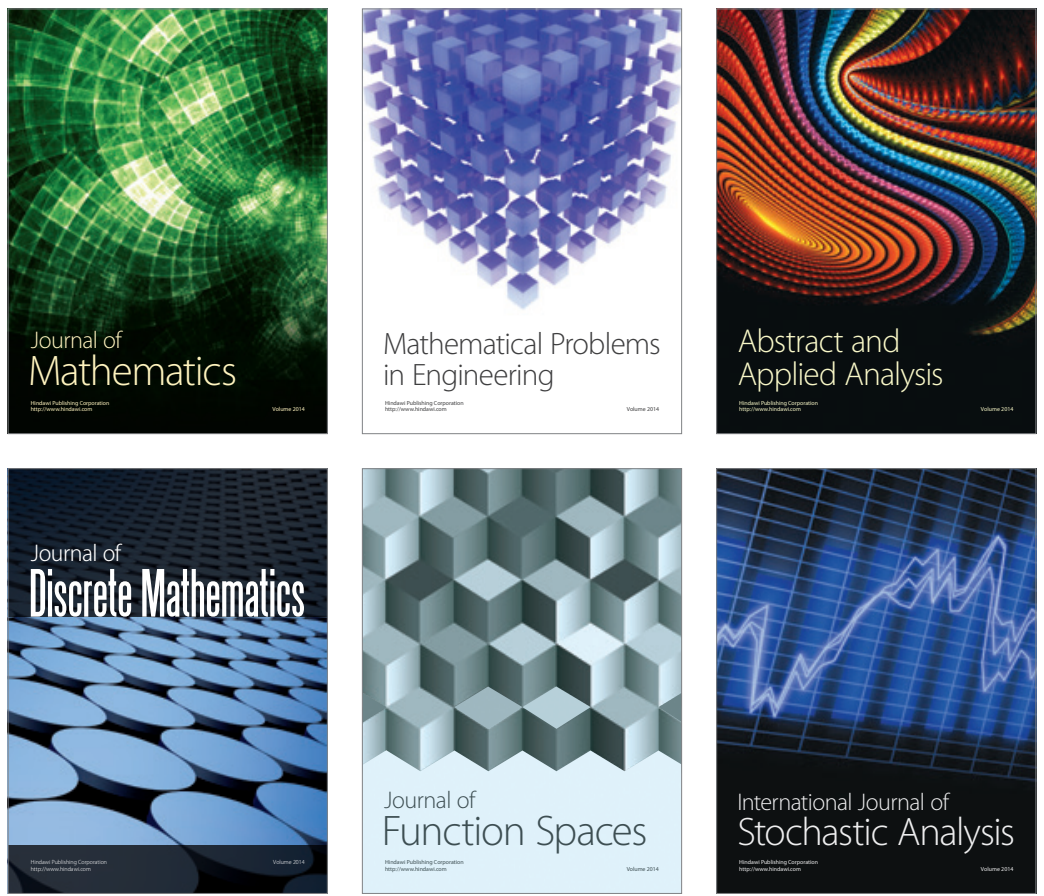

Journal of

Function Spaces

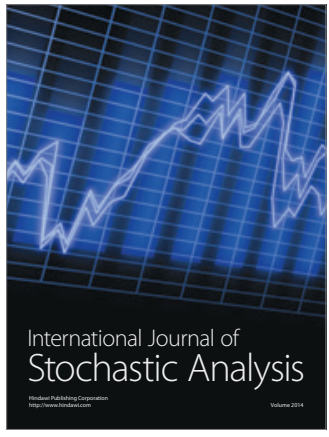

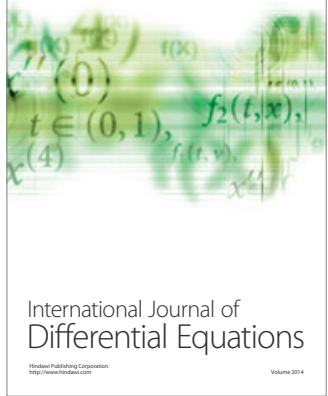
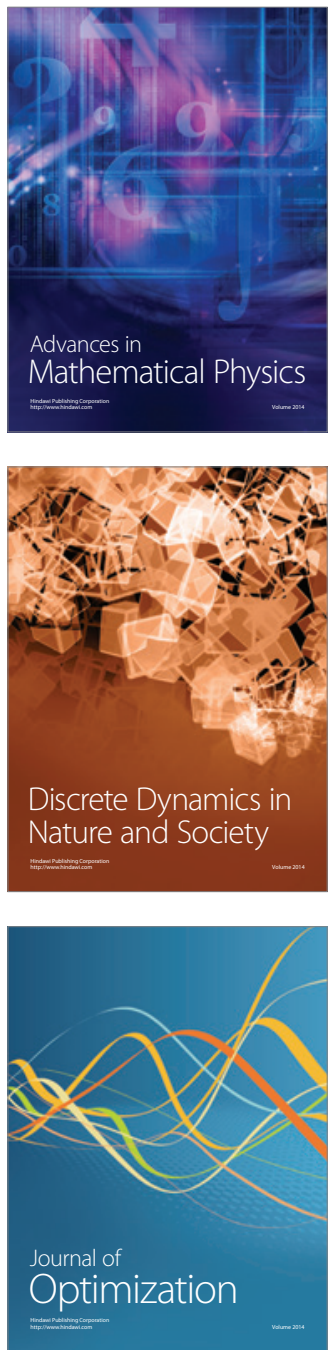